\title{
ASIMETRÍA TEMPORAL Y PARTÍCULAS ELEMENTALES
}

\author{
CRISTIAN ARIEL LÓPEZ \\ Universidad de Buenos Aires / Université de Lausanne / CONICET, ARGENTINA \\ lopez.cristian1987@gmail.com
}

\begin{abstract}
The aim of this article is to argue that a temporal asymmetry may be established within the framework of quantum field theory, independently of any violation of CP, and thereby $\mathrm{T}$, in weak interactions, and independently of the property of time reversal invariance that its dynamical equations instantiate. Particularly, I shall argue that the temporal asymmetry can be stemmed from assessing the links between the proper group of symmetries of the theory and the ontology of the theory: arguments applied to establish which elements and magnitudes remain invariants under group transformations can also be used to establish a temporal asymmetry.
\end{abstract}

Keywords: Philosophy of physics $\bullet$ arrow of time $\bullet$ quantum field theory $\bullet$ symmetry group • neutral kaon.

ACCEPTED: 05/02/2019

\section{Introducción}

El tiempo parece exhibir la propiedad de asimetría: si dos sucesos no son simultáneos, entonces uno es anterior al otro, es decir, existe entre ellos una relación asimétrica. Pero, ¿cuál es la naturaleza de esta asimetría? Además, asumimos que el tiempo posee un carácter unidireccional manifiesto: ¿es esta direccionalidad privilegiada una propiedad esencial del tiempo mismo o, simplemente, una apariencia empírica del nivel macroscópico? Las investigaciones en el contexto de la filosofía de la física buscaron encontrar una asimetría material como correlato y fundamento de la asimetría temporal manifiesta, permitiendo - al menos en principio - un abordaje del problema de la flecha del tiempo a partir del andamiaje formal y conceptual de nuestras teorías físicas. Pero, ¿recogen éstas una dirección temporal?

Desde sus inicios, las líneas de investigación abocadas a responder este interrogante encontraron una dificultad. Por un lado, se reconocen procesos físicos macroscópicos que parecen distinguir una dirección del tiempo e, incluso, leyes físicas, como el segundo principio de la termodinámica, que resultan temporalmente asimétricas, es decir, que no describen los fenómenos de la misma manera en ambos sentidos temporales . Sin embargo, la microfísica y sus leyes fundamentales tienen la propiedad de permanecer invariantes ante inversión temporal (i.e. resultan $T$-invariantes) 
sugiriendo que no son capaces de distinguir entre la dirección pasado-futuro y la dirección futuro-pasado. Este problema enfrenta el ámbito macro-físico con el ámbito micro-físico y adquiere tal relevancia que en la bibliografía al respecto se ha asumido que el problema de la flecha del tiempo depende de reconciliar las disímiles propiedades respecto del tiempo de los dos ámbitos (cf. North 2012, Wallace 2012). Pero, ¿es tal reconciliación posible?

Este problema en particular ha sido, tradicionalmente, abordado a partir del estudio de las propiedades formales de las leyes fundamentales de la física: se supone que si las leyes fundamentales de nuestras mejores teorías físicas son $T$-invariantes, deberíamos concluir que la física no recoge en su formalismo una direccionalidad privilegiada del tiempo en su nivel fundamental, esto es, que la estructura temporal asumida por las diferentes teorías es simétrica. En otras palabras, la $T$-invariancia de las leyes físicas fundamentales implicaría que la asimetría temporal que percibimos es una mera apariencia sin sustrato físico formal.

Discusiones recientes en el ámbito de la filosofía de la física han dado un nuevo giro en la búsqueda de una fundamentación física de la asimetría temporal. Una respuesta plausible al problema de la flecha del tiempo provendría del particular comportamiento de ciertas partículas elementales. Se ha argumentado (Maudlin 2002, North 2012) que existe una asimetría temporal fundamental en la física de partículas, la cual demostraría que la física fundamental no es indiferente a la dirección del tiempo (Maudlin 2002, p.267): la violación de la simetría CP (simetría de carga y de paridad, respectivamente) en interacciones débiles (en particular, por el comportamiento asimétrico del decaimiento de kaones neutros), implica la violación de $\mathrm{T}$ (la simetría temporal), dada la vigencia del teorema CPT en teoría cuántica de campos (marco teórico de la física de partículas actual). Más allá de la plausibilidad o no de una flecha del tiempo fundada en las interacciones débiles, el giro en la discusión tiene un resultado adicional más que interesante: el terreno de los argumentos se desplaza de sus contextos clásicos (como la termodinámica, la mecánica estadística o la relatividad general) a la teoría cuántica de campos (en adelante, TCC).

En el presente trabajo argumentaré TCC provee los suficientes recursos conceptuales y formales para establecer una asimetría temporal no convencional al interior de la teoría, lo que permitiría definir una flecha del tiempo. Estos motivos son independientes de que, por un lado, la teoría sea, efectivamente, invariante ante inversión temporal y, por el otro, de las violaciones de simetría temporal en interacciones débiles. Con respecto al primer punto, aunque la teoría sea estricta y técnicamente invariante ante inversión temporal, sí tiene un medio interno y teórico (no meramente factual y arbitrario) de distinguir ambas direcciones temporales; y el argumento para establecer este punto proviene del análisis del grupo de simetrías de la teoría y la ontología de la teoría. Con respecto al segundo punto, el argumento que presentaré es independiente de cualquier violación de simetría temporal que involucre el 
teorema CPT, tal como sucede con la violación de CP en interacciones débiles.

Con vistas a desarrollar los argumentos, el trabajo se articulará de la siguiente manera. En la Sección 2, formularé de manera general en qué consiste el problema de la flecha del tiempo en la filosofía de la física y cómo se introduce TCC en la discusión. A continuación, en la Sección 3, presentaré de manera general y conceptual TCC, poniendo el acento en el desarrollo del grupo de simetría propio de la teoría (el Grupo de Poincaré) y su relación con el estatus ontológico de las partículas, siguiendo el análisis de Eugene Wigner. En la Sección 4, expondré mis argumentos para establecer una asimetría temporal en TCC a la luz de lo dicho en la Sección 3. Finalmente, brindaré algunas conclusiones con algunas líneas de investigación futuras.

\section{El problema de la flecha del tiempo en la filosofía de la física}

¿Qué significa que el tiempo tiene una direccionalidad? ¿Existe una diferencia sustancial entre el pasado y el futuro? Desde el punto de vista de nuestras intuiciones cotidianas, adjudicamos al pasado ciertas propiedades que no adjudicamos al futuro: mientras que consideramos que el pasado permanece fijo e inalterable, el futuro se presenta como mera posibilidad, indeterminado y abierto. Además, consideramos que el tiempo posee una dirección privilegiada, en la medida en que los sucesos van de pasado a futuro, pero no a la inversa. Desde esta perspectiva, la respuesta parece evidente: el tiempo sí parecería tener la propiedad de asimetría y la propiedad de tener una dirección privilegiada.

$\mathrm{Si}$, como ha sostenido David Hume, la investigación filosófica no es sino la reflexión sobre nuestras ideas e impresiones cotidianas, podemos preguntar: ¿tiene el tiempo, realmente, estas propiedades? Al enfatizar la palabra "realmente", se sugiere que la justificación debería ir más allá de lo que intuitivamente creemos. En otras palabras, uno espera que tales creencias o impresiones respecto de la temporalidad tengan una justificación en alguna propiedad del mundo. Y si suponemos que es la física quien describe de manera más adecuada las propiedades de nuestro mundo, entonces uno esperaría que tales propiedades, de ser reales y objetivas, ${ }^{1}$ sean capturadas y descriptas por alguna de nuestras mejores teorías físicas actualmente vigentes.

Si bien las aproximaciones al problema de la flecha del tiempo o de la asimetría temporal ${ }^{2}$ podrían ser múltiples, desde la psicología cognitiva hasta la biología, pasando por la filosofía del lenguaje, el abordaje desde la filosofía de la física tiene un privilegio epistemológico por sobre el resto. Por un lado, el tiempo es una variable fundamental presente en la mayoría de las leyes físicas e, incluso, es objeto de estudio de algunas teorías físicas (v.g. la relatividad general). Por otro lado, la discusión en 
torno al problema de la flecha del tiempo se ha desarrollado, casi exclusivamente, en el terreno de la física y la filosofía de la física. La manera de formular el problema en las discusiones filosóficas y científicas al respecto, y también sus posibles respuestas, están impregnadas de argumentos provenientes de la física y la filosofía de la física.

\subsection{Asimetría física y flecha del tiempo: irreversibilidad y T-invariancia}

Abordar el problema desde la física y la filosofía de la física nos conduce a prestar atención a las teorías físicas vigentes y atender a qué tipo de relación puede establecerse con la flecha del tiempo. La estrategia consiste en encontrar alguna característica material del mundo que pueda ser coordinada de una u otra manera con la direccionalidad temporal (Sklar 1974, p.355), es decir, en tratar de reflejar en el formalismo o en el contenido de alguna teoría física vigente la idea de un tiempo asimétrico. Pero, ¿rescatan las teorías de la física estas propiedades del tiempo? En caso de hacerlo, ¿cómo lo harían?

Supongamos que hay un vaso sobre la mesa. El vaso, en un momento determinado, cae al piso y se rompe en numerosos pedazos. Quien entra a la habitación puede, rápidamente, reconstruir qué ha sucedido en la habitación: había un vaso sobre la mesa que, luego, cayó al piso y se rompió. Nunca hemos visto la escena temporalmente inversa: un vaso roto en el piso que, espontáneamente, se reconstruye y se ubica en la mesa de manera intacta, con cada molécula de agua en su interior. Llamamos a esta clase de procesos macro físicos, irreversibles.

Ahora bien, podemos describir esta misma situación desde el punto de vista de la física recurriendo a la estructura formal y conceptual de la mecánica estadística clásica. El vaso con agua es ahora una abrumadora cantidad de partículas que están dispuestas espacialmente de una manera particular, donde cada partícula, en principio, tiene una posición y velocidad determinada en el instante $t_{0}$ - que corresponde a lo que macroscópicamente vemos como un vaso sobre la mesa. En un determinado momento, las partículas dejan de estar confinadas en el espacio definido por el vaso y comienzan a alejarse a distintas velocidades, cambiando radicalmente su disposición espacial: las partículas han cambiado su posición, su velocidad y, ahora, si las tomamos en conjunto, ocupan un espacio completamente diferente, encontrándose ampliamente dispersas. Este instante, $t_{1}$, corresponde a lo que macro físicamente veíamos como numerosos pedazos de vidrio y agua sobre el piso. En principio, podríamos describir la trayectoria de cada partícula según las leyes de la mecánica clásica y determinar una evolución particular del sistema en la cual, en un principio, las partículas se encontraban en un cierto estado y, luego, al cabo de un tiempo, las encontramos en otro estado diferente. Nada hay de extraño allí y el mundo macro físico encuentra en la descripción del mundo micro físico una lupa sumamente precisa, acompañado de un formalismo en sintonía con lo observable. Llamemos a esta situación $S_{1}$. 
Sin embargo, todavía mirando el mismo fenómeno pero desde el punto de vista micro físico supongamos una situación distinta, a saber, $S_{2}$ : tomamos el conjunto de partículas que encontrábamos en $t_{1}$ e invertimos todas las variables definidas en función de la variable $t$ (v.g. sus velocidades). Las partículas, espontáneamente, comienzan a reagruparse, hasta llegar a ocupar una distribución espacial muy particular: todas ellas dentro del vaso. Claramente quedaríamos perplejos ante esta evolución. Pero tomemos, ahora, una partícula sola entre la abrumadora cantidad de partículas en cuestión y hagamos la misma operación. Simplemente, determinaremos la trayectoria de una partícula que primero está en un lugar en un cierto estado y al cabo de un tiempo se encuentra en otro estado y otro lugar. Nada de extraño hay en ello. Pero, entonces, no debería haber nada de extraño si consideramos un número mayor de partículas ni motivos para aplicar un razonamiento distinto si consideramos una partícula o si consideramos una cantidad de $10^{23}$ partículas: las leyes de la mecánica clásica que describen a una partícula describen a todas de la misma manera. Por lo tanto, desde el punto de vista del formalismo físico que describe tanto a $S_{1}$ como a $S_{2}$, nada hay de sorprendente: $S_{2}$ es una evolución tan posible como $S_{1}$ dado el formalismo de la mecánica estadística clásica.

Comencemos a precisar los términos. Llamamos T-invariancia a la propiedad de las leyes físicas de ser invariantes ante inversión temporal, e irreversibilidad a la propiedad que tienen los fenómenos de evolucionar en una dirección temporal y nunca en la contraria.

Definición 1 Una ecuación dinámica es T-invariante si es invariante bajo la aplicación del operador de inversión temporal $T$, el cual lleva a cabo la operación $t \rightarrow-t$ e invierte todas las variables dinámicas definidas en función de $t$. Como resultado, si e $(t)$ es una solución a la ecuación dinámica, Te $(t)$ es también una solución. (Castagnino y Lombardi 2009, p.3)

El concepto de $T$-invariancia, como vemos, es una propiedad de las leyes físicas, que depende de la particular forma matemática de la ecuación dinámica que la expresa. Una gran cantidad de leyes físicas tienen la propiedad de ser $T$-invariantes: las leyes de la mecánica de Newton, las ecuaciones de campo de Einstein, las leyes de la mecánica cuántica no relativista y relativista, las ecuaciones de Maxwell, entre otras. Nótese que las soluciones $e(t)$ y $T e(t)$ de ecuaciones $T$-invariantes constituyen un par simétrico (en adelante, par $T$-simétrico), tal como concebíamos las situaciones $S_{1}$ y $S_{2}$. La existencia de pares $T$-simétricos es lo que desdibuja cualquier distinción entre ambas direcciones temporales.

Definición 2 Una evolución es reversible si no tiene estados de equilibrio finales (o iniciales), es decir, "puntos de no retorno" (Castagnino y Lombardi 2009, p.3). 
A diferencia del caso anterior, reversibilidad e irreversibilidad son propiedades que se aplican a las soluciones de las ecuaciones dinámicas (i.e. a las evoluciones) y no a las ecuaciones mismas. En definitiva, $T$-invariancia y reversibilidad se predican de entidades diferentes. Esta diferencia permite mantener en claro el rango de aplicabilidad de ambos términos para evitar posibles confusiones. En general, se ha sostenido que la clave para la solución al problema de la flecha del tiempo radica en el concepto de irreversibilidad; pero, al mismo tiempo, se afirma que es necesario encontrar leyes que permitan recoger una direccionalidad del tiempo (es decir, que sean leyes no $T$-invariantes). Aquí hay, al menos, dos confusiones: o bien no hay una clara y precisa distinción entre las propiedades de reversibilidad y $T$-invariancia, y se usan indistintamente sin atender que corresponden a propiedades de entidades matemáticas distintas; o bien se presupone que existe una correlación entre leyes $T$-invariantes y evoluciones reversibles y que allí donde hay procesos irreversibles hemos de encontrar leyes no $T$-invariantes que los describan. Esto constituye una confusión dada las Definición 1 y la Definición 2, y porque ambas propiedades admiten, físicamente, todas las combinaciones posibles: existen procesos irreversibles descriptos por leyes $T$-invariantes, como leyes no $T$-invariantes que cuyas soluciones son reversibles (cf. Castagnino y Lombardi 2005, pp.75-6).

Hay además una diferencia de carácter modal entre ambos conceptos. Mientras que el concepto de $T$-invariancia se predica propiamente de las leyes físicas $\mathrm{y}$, consecuentemente, del conjunto de todas sus soluciones, el concepto de reversibilidad/irreversibilidad se predica de una solución en particular. Que una ley sea $T$-invariante indica, básicamente, que por cada evolución posible de acuerdo a la ley existe otra evolución posible (un mundo posible de acuerdo a la ley) cualitativamente igual a la original pero con la dirección temporal invertida; esto permite establecer el rango de modelos posibles de acuerdo a la teoría. Si la ley resulta no- $T$-invariante, la teoría no tiene modelos en ambas direcciones temporales, sino sólo en uno. Es decir, no es posible de acuerdo a la teoría dos mundos cualitativamente iguales pero con direcciones temporales invertidas: sólo uno de ellos es posible. Sin embargo, la noción de reversibilidad es mucho más acotada, circunscribiéndose a las propiedades de una evolución en particular (por ejemplo, la historia de nuestro universo): nada dice acerca de la posibilidad o imposibilidad de otras evoluciones de acuerdo a la teoría, ni de la estructura modal de la teoría o el conjunto de sus modelos.

\subsection{A la búsqueda de leyes no T-invariantes: ¿un resultado negativo?}

En términos generales, el problema de la flecha del tiempo se ha abordado en términos de procesos físicos irreversibles, como aquellos sistemas termodinámicos que experimentan un aumento de su entropía hasta alcanzar el equilibrio al estar aislados. De acuerdo a lo expuesto en la sección anterior, procesos irreversibles como 
estos señalarían que una evolución en particular selecciona una dirección privilegiada del tiempo. Sin embargo, tal evolución podría ser uno de los miembros de un par $T$-simétrico, ya que podría ser solución de una ecuación de movimiento $T$-invariante. Querer fundamentar una direccionalidad temporal (en un sentido robusto) en base a procesos irreversibles parece no sólo ocultar la existencia de una evolución temporalmente invertida que es solución de la misma ecuación, sino - y en virtud de ello - cierta circularidad: cuando consideramos que la entropía aumenta hasta lograr el equilibrio hacia el futuro pero nunca aumenta hacia el pasado, estamos presuponiendo la asimetría temporal ya que localizamos el equilibrio en el futuro, cuando podríamos igualmente ubicarla en el pasado si en su lugar consideráramos al otro miembro del par $T$-simétrico. Este tipo de asimetrías temporales en términos de procesos irreversibles (como incremento de la entropía o expansión del universo) no parecen ser lo suficientemente fuertes para establecer una asimetría del tiempo mediante mecanismos no arbitrarios, y en muchos casos meramente señalan una asimetría de cosas en el tiempo (Savitt 1996, p.348, North 2012, p.312).

Lo relevante en la discusión es determinar si existe una flecha del tiempo que pueda establecerse de manera no arbitraria, no circular y recurriendo a elementos internos de la teoría. A fin de cuentas, es la teoría misma la que tiene que disponer de los recursos necesarios que nos permitan seleccionar una dirección temporal y descartar la opuesta. Trivialmente, siempre es posible romper una simetría temporal con elementos externos a la teoría, pero no es eso lo que está en juego aquí.

Una estrategia usual para identificar una asimetría temporal a partir del formalismo mismo de la teoría, y que daría fuertes indicios de una asimetría fundamental del tiempo, es en términos de leyes no- $T$-invariantes. Una ley tal, por definición, no generaría un par de gemelos $T$-simétricos sino que sólo produciría soluciones en una dirección temporal pero no en la contraria. Para decirlo de otra manera, la teoría no tendría modelos en ambas direcciones temporales, sino sólo en uno. Si bien nada en la ley nos señalaría qué dirección es el futuro o el pasado (pues esto es una cuestión puramente nominal), sí nos señalaría una diferencia sustancial que, convencionalmente, podríamos bautizar como la dirección pasado-futuro. El argumento desde esta estrategia podría esquematizarse de la siguiente manera:

i. Si existen leyes de la física que sean no $t$-invariantes, entonces hay argumentos físicos para establecer la flecha del tiempo.

ii. Existe una ley física que es no $t$-invariante.

iii. Ergo, hay argumentos físicos para establecer la flecha del tiempo.

Nótese que la premisa fundamental es la segunda, y que la primera es el supuesto necesario para formular este argumento de tipo nomológico. La verdad o no de la segunda premisa es una cuestión que radica, esencialmente, en las propiedades 
formales de las teorías físicas fundamentales actualmente vigentes.

Vale la pena contrastar un caso de no- $T$-invariancia en las leyes, con un caso de irreversibilidad en una solución: mientras que en este último caso una solución en particular resulta irreversible (presentando las características mencionadas en el apartado anterior), y es compatible con soluciones también irreversibles pero temporalmente invertidas, el caso de leyes no- $T$-invariantes implica que cualquier solución de la teoría tendrá sólo una dirección temporal, y que cualquier evolución temporalmente invertido es imposible (es decir, no es una solución) de acuerdo a la teoría. El punto es que, tal como se asume comúnmente en física teórica, las simetrías espacio-temporales de una teoría permitirían ciertas inferencias respecto del tipo de estructura espacio-temporal asumido por la teoría: si la dinámica de la teoría resulta ser invariante ante traslación espacial, uno podría deducir que la teoría asume que el espacio es homogéneo (ver North 2009). El mismo razonamiento es aplicable a la simetría ante inversión temporal: si las ecuaciones de movimiento de una teoría son $T$-invariantes, entonces la estructura temporal de la teoría es simétrica.

Es comprensible que el tema de la $T$-invariancia o no $T$-invariancia de las leyes de la física haya ocupado una enorme atención en las discusiones sobre la temporalidad en física dado que resulta una estrategia rotunda para establecer una flecha del tiempo. Tim Maudlin afirma al respecto:

El tratamiento de esta cuestión es uno de los más peculiares en la literatura filosófica. El enfoque usual configura el problema como sigue: las leyes físicas fundamentales tiene una característica llamada invariancia ante inversión temporal. Si las leyes son invariantes ante inversión temporal, entonces se supone que se sigue que la física misma no reconoce una direccionalidad del tiempo: no distingue, a nivel de ley fundamental, la dirección hacia el futuro de la dirección hacia el pasado. (Maudlin 2007, p.266)

David Wallace presenta el mismo diagnóstico que Maudlin sobre las leyes fundamentales de la física actual:

En la mayoría de las teorías [físicas] relevantes para nosotros, podemos hallar un operador de inversión temporal que deja invariante la energía de un sistema, la densidad de masa y, esencialmente, toda propiedad de relevancia macroscópica, observable de un sistema. (Wallace 2012, p.265)

Cabe señalar que existen objeciones acerca de si la característica de $T$-invariancia de las leyes fundamentales les pertenece realmente a las leyes físicas; es decir, existen objeciones que apuntan a mostrar que la $T$-invariancia no es una verdadera propiedad de las leyes (Albert 2001, Capítulo 1). Sin embargo, en general se acuerda que las leyes fundamentales (como las leyes de la mecánica clásica, las ecuaciones de campo de la relatividad general y las leyes de la mecánica cuántica no relativista) tienen de 
hecho la propiedad de $T$-invariancia. Ni la mecánica clásica, ni la relatividad general, ni la mecánica cuántica no relativista y relativista ofrecen una estructura formal capaz de hacer verdadera la segunda premisa del argumento anterior, es decir, capaz de distinguir nomológicamente los procesos físicos que evolucionan hacia el pasado de los que evolucionan hacia el futuro.

Uno podría preguntarse si leyes no- $T$-invariantes son necesarias para establecer una flecha del tiempo en física. Esta pregunta conduce a cuestionar cuál es el alcance de la primera premisa del argumento. Tal como fue formulado, parece que el requisito de no- $T$-invariancia es una condición suficiente, pero no necesaria, para una flecha del tiempo en sentido sustantivo. Ahora bien, ies posible formular un tipo similar de argumentación sin la necesidad de disponer de leyes no- $T$-invariantes? Es importante tener claro que lo que uno tiene que lograr para establecer una flecha del tiempo en sentido sustancial es poder escoger, de manera no arbitraria y mediante recursos de la propia teoría, un conjunto de soluciones del par de gemelos $T$-simétricos. Esto podría sugerir que si bien la $T$-invariancia es una condición suficiente, podría no ser necesaria: podría haber alguna estrategia alternativa para establecer una asimetría y dirección temporal en una teoría determinada a pesar del carácter $T$-invariante de sus ecuaciones fundamentales. Claramente, tal estrategia alternativa no implicaría que la estructura temporal de la teoría es en sí misma asimétrica (ya que esto sólo podría ser garantizado por leyes no- $T$-invariantes). Pero creo, sin embargo, que tal requisito no es necesario para el problema de cómo seleccionar de manera no arbitraria un par del gemelo $T$-simétrico y, por lo tanto, establecer una asimetría y direccional temporal en un sentido robusto. Una teoría $T$-invariante pero con los recursos conceptuales y formales adecuados para descartar uno de los miembros del par $T$-simétrico establecería igualmente una flecha del tiempo sustantiva.

Mi argumento en este trabajo se basa en una instancia de este tipo de estrategia: TCC es una teoría $T$-invariante pero con recursos teóricos adicionales que permiten romper la simetría temporal de manera no arbitraria. Se ha argumentado que el decaimiento asimétrico de kaones neutros podría ser un indicio de una asimetría temporal fundamental. Analizaré este caso en la subsección siguiente. En la Sección 4, daré mi argumento alternativo para una asimetría temporal fundamental en TCC que no recurre ni a una presunta no- $T$-invariancia de TCC, ni al particular decaimiento de kaones neutros.

\subsection{Flecha del tiempo y teoría cuántica de campos: el decaimiento del kaón neutro}

Algunos autores han focalizado en ciertos procesos físicos microscópicos descriptos por la teoría cuántica de campos (en adelante, TCC) de los cuales se podría inferir la existencia de, al menos, una violación de la simetría temporal y, por lo tanto, de una 
asimetría temporal fundamental. En este sentido, Jill North sostiene:

Tenemos, ahora, evidencia experimental de que hay una asimetría temporal legal y fundamental en nuestro mundo. Dado el teorema CPT, la violación de paridad observada en el decaimiento del mesón $K_{0}$ (neutral, sin carga) implica la violación de la simetría temporal. (North 2012, p.315)

Según el teorema CPT, cualquier teoría cuántica de campos (lorentziana) debe ser CPT-invariante, esto es, invariante frente a la combinación de conjugación de carga, inversión de paridad e inversión temporal. Por lo tanto, la violación de la conjugación de la carga (C) o de la inversión de la paridad (P) implica la ruptura de la simetría temporal (T). Hay quienes han leído esta violación de la simetría temporal como una genuina asimetría temporal.

Yo no estoy muy seguro que esta violación de la simetría temporal al nivel de las interacciones débiles sea una asimetría temporal fundamental. En primer lugar, como se mencionó más arriba, la teoría física de marco para describir estos procesos es TCC cuyas ecuaciones dinámicas son invariantes ante la acción de invertir el tiempo; en el caso de los decaimientos, la $T$-invariancia de TCC sigue rigiendo, sólo que se dispondría - de acuerdo a mi punto de vista — de evidencia adicional para establecer que esa simetría temporal regida por la dinámica fundamental de la teoría incluye casos de soluciones irreversibles.

De todas maneras, el asunto es por demás interesante. No sólo la llave para resolver el problema de la flecha del tiempo con argumentos físicos parece estar al alcance de la mano, sino que la introducción de un nuevo ámbito de discusión, como es TCC, se presenta como un poderoso avance en el tratamiento del problema, abriendo nuevas puertas, nuevas estrategias y nuevas discusiones. Ya sea impugnando la legitimidad del teorema CPT en el contexto de la discusión (Horwich 1987) o argumentando en favor de su legitimidad (Maudlin 2007, Wallace 2012), ya sea mostrando la insuficiente evidencia empírica al respecto (Sachs 1987), o discutiendo cuál es la naturaleza del teorema CPT y su relación con el tiempo (Arntzenius 2012, Greaves 2010), TCC se ha convertido, indudablemente, en un ámbito de discusión sobre el problema de la flecha del tiempo por derecho propio.

Incluso aun suponiendo que la violación de $\mathrm{CP}$ y por lo tanto de $\mathrm{T}$ en interacciones débiles sea una genuina asimetría temporal a nivel de la física fundamental, considero que existe un criterio previo y, a mi juicio, más fundamental para establecer una asimetría temporal, a pesar del carácter estrictamente $T$-invariante de las ecuaciones dinámicas de la teoría, y a pesar de que la estructura espacio-temporal supuesta por la teoría sea temporalmente simétrica. El criterio que presentaré se basa, principalmente, en analizar el grupo de simetrías propio de la teoría, sus representaciones irreducibles y qué tipo de estados y observables que la teoría considera que tienen significado físico. El criterio es más fundamental porque recurre a principios 
generales de la teoría (axiomas en sus formulaciones axiomáticas) que circunscriben su ontología y su relación con las posibles evoluciones temporales. Estos elementos, como mostraré, permiten establecer una asimetría temporal ya que permiten de manera no arbitraria seleccionar sólo un miembro del par de gemelos $T$-simétricos de manera unívoca.

\section{Teoría cuántica de campos: grupo de simetría y ontología}

Aunque no es un teorema, se cree, ampliamente, que es imposible reconciliar la mecánica cuántica y la relatividad, excepto en el contexto de la teoría cuántica de campos. (Weinberg 1987, pp.78-9)

Si bien las palabras de Weinberg respetan fielmente el espíritu de lo que TCC pretende ser, qué es TCC no es una pregunta fácil de responder. A diferencia de otras teorías físicas, carece de una definición canónica y universalmente aceptada (cf. Kuhlmann 2012). Ni siquiera existe un acuerdo medianamente consensuado acerca de qué habla TCC, es decir, cuáles son las entidades y estructuras a las cuales la teoría refiere. La teoría, ¿da prioridad ontológica a las partículas o a los campos?, ¿qué son las partículas?, ¿qué significa que un campo ha sido cuantificado? Incluso peor, TCC no puede garantizar que todas sus representaciones canónicas sean equivalentes (como la mecánica cuántica estándar), por lo que habría una multiplicidad de teorías cuánticas de campo. Mi intención aquí no es presentar un análisis exhaustivo de TCC o argumentar en favor o en contra de una interpretación en particular, sino sentar las bases de una comprensión que establezca algunos parámetros generales para hablar de TCC. Por ello, tomaré una vía más epistemológica: la relación de TCC con las dos teorías físicas más cercanas, la mecánica cuántica y la relatividad especial (en adelante, MC y RE respectivamente).

Una manera de entender el estatus teórico de TCC en la física contemporánea es entenderla como la teoría que ofrece un marco teórico unificado para las teorías físicas fundamentales (Kuhlmann, Lyre y Wayne 2002, p.5), ya que permite el tratamiento de partículas y campos dentro de un marco teórico común. TCC sería la teoría resultante de reconciliar MC con RE mediante la cuantización del campo relativista o, en otras palabras, la extensión de la MC a campos, i.e., sistemas con infinitos grados de libertad. En efecto, por un lado, TCC puede entenderse como una extensión de MC ya que permite la descripción de sistemas con un número variable de partículas (Kuhlmann 2012); por otra parte, TCC puede entenderse como el intento de aplicar la covariancia relativista a MC.

Sin embargo, esta manera de intentar esclarecer qué es TCC mediante sus relaciones con MC y RE tiene sus límites (Kuhlmann 2012): existen versiones de TCC de carácter no relativista (cf. Bain 2011) como también inconsistencias teóricas a la hora 
de poner en conjunción RE con MC. La oscuridad interpretativa alrededor de TCC es evidente. Sin embargo, creo que lo dicho hasta el momento permite entender a qué nos estamos refiriendo de manera general cuando hablamos de TCC o, al menos, qué papel ocupa TCC en relación a las otras teorías físicas contemporáneas.

En esta sección, avanzaré sobre dos puntos — íntimamente relacionados - , los cuales consideraré como fundamentales para comprender el problema de la flecha del tiempo en el contexto de TCC: la célebre interpretación y definición de partícula ofrecida por Eugene Wigner en 1939 y los conceptos de simetría y grupo de simetría, en particular, el Grupo de Simetría de Poincaré. Estos elementos serán necesarios para, en la Sección 4, analizar TCC en relación con el problema de la flecha del tiempo.

\subsection{La relación entre simetría y ontología}

El texto de Eugene Wigner, "On unitary representations of the inhomogeneous Lorentz group" (1939), intenta brindar una definición rigurosa de qué es una partícula. La idea general, filosóficamente interesante, que está detrás del planteo de Wigner es la conexión entre el grupo de simetría físicamente relevante de la teoría con la ontología de la teoría.

En un primer acercamiento, el concepto de simetría refiere a una relación de proporción cuya función consiste en armonizar los diferentes elementos en una totalidad unitaria donde las partes son intercambiables con respecto al todo (Brading y Castellani 2017). Probablemente, las simetrías más intuitivas sean las simetrías espaciales bilaterales, y rotacionales. Sin embargo, la definición moderna de simetría (tal como surgió en el siglo diecisiete en el ámbito de las matemáticas) se entiende en función del concepto de invariancia que es más general y permite su aplicación a expresiones matemáticas diversas (e.g. ecuaciones de movimiento). En estos términos, simetría es la invariancia bajo un grupo específico de transformaciones. Más formalmente, sea un conjunto $X$ de objetos $x_{i} \in X$, y un grupo $G$ de transformaciones $g_{a} \in G$, donde las transformaciones $g_{a}: X \rightarrow X$ actúan sobre los objetos $x$ como $x_{i} \rightarrow x$. Un objeto $x_{i} \in X$ es invariante ante la transformación $g_{a}$ si, para tal transformación, $x_{i}=x$; a su vez, $x_{i} \in X$ es invariante ante el grupo $G$ si es invariante ante todas las transformaciones $g_{a} \in G$. Tales transformaciones pueden ser entendidas en términos pasivos (v.g. transformar el sistema de referencia desde el cual se describe al sistema) o en términos activos (donde se transforman todos los objetos físicos involucrados en el sistema). En virtud de esta noción abstracta de simetría, los distintos elementos quedan relacionados unos con otros y con la totalidad, de modo que forman una unidad con una cierta regularidad. Tal regularidad del todo emerge sobre la base de la naturaleza específica del grupo de transformaciones (para más detalles, ver Brading y Castellani 2007). 
Cada teoría física tiene un grupo de simetría que le es propio, es decir, un conjunto de transformaciones que mantiene invariante la estructura de las leyes de la teoría (Kuhlmann 2002, p.87). La idea que está detrás de esta forma de entender la simetría en términos de teoría de grupos y su relación con la ontología de una teoría física consiste en que las operaciones de simetría cambian la perspectiva del observador pero no modifican las propiedades mismas de los sistemas físicos. Intuitivamente, cuando pretendemos describir un objeto físico determinado, no pensamos que el objeto cambia por moverlo de una habitación a otra, por rotarlo o por verlo desde una perspectiva distinta. Se trata del mismo objeto. La noción de grupo de simetría busca rescatar esta idea: analizar el grupo de simetría físicamente relevante de una teoría nos informa acerca de qué objetos permanecen invariantes ante su conjunto de transformaciones específico. Y es natural que ésas sean precisamente los objetos privilegiados y fundamentales de la teoría en cuestión, que luego recibirán una interpretación ontológica determinada.

\subsection{TCC y el Grupo de Poincaré ${ }^{3}$}

Mencioné al principio de la sección que una de los modos de pensar qué es TCC consiste en considerarla en relación con RE: al ser TCC una teoría que introduce la invariancia relativista, el grupo de simetría relevante de la teoría es el Grupo de Poincaré, que es el grupo de invariancia correspondiente al espacio-tiempo cuadridimensional de Minkowski. Por este motivo se dice que el Grupo de Poincaré es el grupo de simetría propio de TCC (denotado por $\left[P_{+}\right]$), ya que la física (de TCC) no cambia bajo las transformaciones especificadas por este grupo. En términos más formales, un elemento del Grupo de Poincaré es una transformación $g=(\Lambda, a)$ actuando sobre un espacio-tiempo de Minkowski, donde $\Lambda$ refiere a las transformaciones de Lorentz (i.e. rotaciones y boosts) y a denota traslaciones en el espacio-tiempo.

Las transformaciones del Grupo de Poincaré actúan directamente sobre sistemas de referencia en el espacio-tiempo de Minkowski (es decir, se trata de simetrías pasivas). Pero, puede establecerse una correspondencia entre este tipo de transformaciones con otras que actúan sobre los estados físicos del sistema (es decir, con simetrías activas): las transformaciones de simetría pueden ser representadas por operaciones sobre el conjunto de estados. Según la teoría matemática de la representación para grupos de simetría, una representación es una técnica utilizada para analizar grupos en términos de operaciones lineales sobre el espacio de vectores. La representación de un grupo estaría dada por el conjunto de operaciones sobre el espacio de estados correspondiente al grupo. El tipo de relación que existe entre el grupo de simetría y su representación en términos de estructuras algebraicas abstractas es un homomorfismo, por lo cual la representación de un grupo preserva la estructura del grupo.

El concepto de representación es central en el análisis de Wigner sobre partículas 
elementales. Precisamente, este concepto permite establecer una relación entre los estados de un sistema físico (una partícula cuántica en el vocabulario de Wigner) y la estructura de un Grupo de Lie (el Grupo de Poincaré es un ejemplo de Grupo de Lie). Todo estado de un sistema cuántico puede representarse como un vector en un espacio de Hilbert (de ahora en adelante, $\mathscr{H}$ ) que representa al sistema. Por lo tanto, el conjunto de vectores en un espacio de Hilbert determinado representa el conjunto de estados posibles para un sistema cuántico. Pero, ¿cualquier vector en $\mathscr{H}$ representa un estado físicamente posible del sistema? Esta es una pregunta fundamental a la hora de determinas cuáles estados son físicamente relevantes y cuáles son superfluos o no tienen ningún significado físico, y una estrategia efectiva para poder determinarlo recurre al concepto de simetría. En $\mathscr{H}$, las entidades matemáticas que representan a las transformaciones del Grupo de Poincaré son operadores unitarios sobre $\mathscr{H}$, formalmente representados como $U(\Lambda, a)$. Estos elementos de la representación del Grupo de Poincaré sobre $\mathscr{H}$ (i.e. los operadores unitarios) permiten la transición $|\psi\rangle \rightarrow\left|\psi^{\prime}\right\rangle=U(\Lambda, a)|\psi\rangle$. Es decir, el operador unitario transforma un estado del sistema en otro.

Naturalmente, el análisis de qué representaciones son posibles y cuáles son sus propiedades conduce a conclusiones acerca de qué vectores son posibles en $\mathscr{H}$ y qué estados representan. La propiedad de irreducibilidad y, en particular, de representación irreducible es el ingrediente clave en el análisis de Wigner sobre el Grupo de Poincaré y su relación con las partículas elementales (Kuhlmann 2010, p.90). Una representación irreducible de un grupo de simetría es una representación del grupo tal que no tiene subespacios invariantes no triviales. En otras palabras, no existe un subconjunto propio de la representación del espacio de estados que sea invariante bajo la acción del correspondiente conjunto de operadores unitarios. ¿Cuál es el significado físico de esta propiedad matemática?, ¿cómo vincularla con las preguntas ontológicas que subyacen al análisis de Wigner de 1939? El punto clave radica en que, de cada representación irreducible, se obtiene el espacio de estados de un sistema físico elemental. Y el análisis de Wigner consiste, en su mayor parte, en ofrecer una clasificación de las representaciones irreducibles del Grupo de Poincaré, es decir, "encontrar magnitudes que sean invariantes relativísticamente y sirvan para clasificar las representaciones irreducibles" (Kuhlmann 2010, p.91).

Consideremos, por ejemplo, el cuadrivector de energía-momento $P^{\mu}$. La magnitud que se conserva bajo transformaciones relativistas es $P^{2}=p^{\mu} p_{\mu}=m^{2} c^{2}$, donde $m$ es la masa y $c$ es la velocidad de la luz. Si, por convención, se adopta $c=1$, se obtiene que el autovalor de $P^{2}$ es $p^{2}=m^{2}$, lo cual constituye el primer parámetro de la clasificación de Wigner. Por otra parte, la componente cero $P^{0}$ del cuadrivector representa la energía, y constituye el segundo parámetro de la clasificación. Ahora bien, ¿cuál es el espectro de valores que $P^{2}$ y $P^{0}$ pueden adoptar? La respuesta a esta pregunta, bajo los supuestos que asume el análisis de Wigner (una ontología de 
partículas), tiene un correlato inmediato en la estructura física y, a su vez, es susceptible de una representación geométrica en un espacio-tiempo relativista. Precisaré este último punto.

Recuérdese que, al encontrarnos en un contexto no galileano, para cualquier evento - supongamos $x_{1}$ - existe una partición del espacio-tiempo de Minkowski en seis regiones (en un contexto galileano tenemos sólo tres: pasado, presente y futuro). En primer lugar, tenemos el punto espacio-temporal en el que se encuentra el evento $x_{1}$. En segundo lugar, la región de los eventos que pueden estar causalmente influenciados por $x_{1}$, es decir, aquellos eventos que se encuentran en el semicono de luz futuro con vértice en el punto espacio-temporal que ocupa $x_{1}$. En tercer lugar, tenemos el semicono de luz pasado: la región de los eventos que puede tener influencia causal sobre $x_{1}$. Esta tripartición recoge las particiones que se pueden llevar a cabo en un contexto galileano, pero es incompleta. Dado que la propagación de señales tiene un límite en RE (dado que no existe señal que viaje a mayor velocidad que la luz), existen límites a la acción causal desde el pasado y hacia el futuro: eventos a los que sólo puede acceder un rayo de luz y eventos que están más allá de lo accesible por un rayo de luz. Estos parámetros nos delimitan tres regiones más en la partición del espacio-tiempo relativista: la superficie del cono de luz futuro, la superficie del cono de luz pasado y la región que permanece fuera de los conos de luz, donde la separación respecto de $x_{1}$ es de tipo espacial.

La clasificación de Wigner busca correlacionar los estados de una partícula y su comportamiento bajo las transformaciones del Grupo de Poincaré con una descripción geométrica en términos del diagrama de Minkowski. Volviendo al caso de $P^{2}$ y $P^{0}$, los valores que tales observables asuman determinarán tanto una interpretación en términos ontológicos (i.e. si representan un estado posible de una partícula cuántica), como una descripción en términos geométricos (ubicando el vector que representa el estado en alguna de las seis regiones del espacio-tiempo de Minkowski). En términos más concretos,

(i) Si $p^{2}=m^{2}>0$ y $p^{0}>0$, entonces la interpretación ontológica $-\mathrm{y}$ estándar - de esta representación irreducible es que se trata de una partícula con masa. En términos geométricos, se ubica en el cono de luz futuro.

(ii) Si $p^{2}=m^{2}>0$ pero $p^{0}<0$, tal clase de representación irreducible no se corresponde con ningún sistema físico posible ya que el rango de los valores del operador de energía sería negativo. La traducción a términos geométricos es particularmente interesante, porque confina a tales estados (vectores) al semicono de luz pasado.

(iii) Si $p^{2}=0$ y $p^{0}>0$, estamos ante partículas sin masa; geométricamente, este caso corresponde a la superficie del cono de luz futura. Por lo tanto, se trata de partículas sin masa que, por ello, pueden moverse a la velocidad de la luz: 
i.e., fotones.

(iv) Si $p^{2}=0$ y $p^{0}<0$, nuevamente estamos ante un caso donde no hay correlato ontológico posible ya que el operador de energía incluiría valores dentro del espectro negativo: se trataría de partículas sin masa pero con energía negativa. En este caso, la descripción geométrica refiere a la superficie del cono de luz pasado.

(v) Si $p^{\mu} \equiv 0$, obtenemos sólo vacío y su representación geométrica como punto.

(vi) Si $p^{2}<0$, entonces estamos ante partículas virtuales de masa negativa. Geométricamente, se corresponden a la región exterior a los conos de luz.

Recapitulemos lo dicho hasta el momento. La intención original era abordar el problema de la flecha del tiempo a partir del análisis de la estructura ontológica de TCC, y la estrategia adoptada consistió en analizar el grupo de simetría propio de la teoría. Utilizamos como guía el análisis de Wigner sobre partículas elementales, el cual nos remitió al concepto de representación irreducible. Finalmente, la clasificación de las representaciones irreducibles del Grupo de Poincaré (grupo de simetría propio de TCC) estableció que algunos estados de los sistemas son estados de partículas con significado físico, mientras que otros estados no tienen significado físico alguno, como por ejemplo, cuando el rango de valores para el operador de energía $P^{0}$ es $p^{0}<0$. Estados con tal rango de energía no son estados de una partícula posible ya que serían altamente inestables. A su vez, estos estados con energías en el rango negativo del espectro, geométricamente quedan confinados en el semicono de luz pasado o en su superficie.

\section{TCC como una teoría temporalmente asimétrica}

Presentado el andamiaje teórico en la sección anterior, es momento de adentrarnos en el problema de la flecha del tiempo en TCC. En esta sección argumentaré que es posible establecer una asimetría temporal en TCC que no depende de fenómenos empíricos sub-atómicos - como el decaimiento del mesón $K_{0}$ en interacciones débiles - ni de la propiedad $T$-invariancia. En cambio, tal asimetría puede establecerse a partir de principios teóricos generales de la teoría que constriñen el tipo de sistemas físicos y estados que TCC tolera en su ontología; sistemas físicos y estados estrechamente relacionadas con la dirección temporal que se escoja.

Sin embargo, TCC es una teoría compleja en muchos sentidos. Para empezar, como se mencionó rápidamente más arriba, TCC es una teoría cuántica de sistemas con infinitos grados de libertad. Esto significa que yace más allá del alcance del teorema de Stone-von Neumann que establece la equivalencia unitaria (y, plausiblemente, física) entre diferentes representaciones de las relaciones de conmutación canónica para un sistema de finitos grados de libertad. Que TCC no se rija por el teorema 
de Stone-von Neumann implica que existen infinitas representaciones unitariamente inequivalentes (y, plausiblemente, físicamente inequivalentes) de sus relaciones de conmutación canónica (ver Ruetsche 2013 para un riguroso análisis de esta característica). Esta situación ya advierte que, en principio, existirían infinitas teorías cuánticas de campos (aplicables a diferentes sistemas de infinitos grados de libertad) que no serían unitariamente equivalentes entre sí.

Una segunda complejidad de la teoría se debe a que existen al menos, y a grandes rasgos, dos versiones: una versión ordinaria o heurística (TCCHe) y una versión axiomática (TCCAx). TCCHe es la versión comúnmente utilizada para explotar el poder predictivo y descriptivo de TCC, aunque renunciando a rigor matemático y tolerando algunas inconsistencias internas. Fue esta falta de rigurosidad y la presencia de inconsistencias la que condujo a formular una versión axiomática de TCC (TCCAx) que establezca de manera clara cuáles eran sus principios básicos, qué se podía deducir de ellos, y que estuviese libre de las inconsistencias de TCCHe. ${ }^{4} \mathrm{Sin}$ alcanzar el grado de poder descriptivo y predictivo de TCCHe, se propusieron dos axiomatizaciones relativamente exitosas: la axiomatización de Wightman (TCCWi) y la axiomatización algebraica de Haag-Kastler (TCCAl). TCCWi y TCCAl son diferentes maneras de depurar TCCHe y ofrecer un marco teórico matemáticamente riguroso, libre de inconsistencias internas y con estructura suficiente para un desarrollo teórico independiente de modelos particulares.

Existe cierta discusión en la filosofía de la teoría cuántica de campos acerca de cuán exitosos fue el proyecto de axiomatizar TCC y cuál son sus relaciones con TCCHe. Aunque el éxito matemático de TCCWi y TCCAl es evidente, el éxito físico, explicativo y predictivo de TCCHe es irrefutable. Algunos han visto en esta última característica un motivo suficiente para declarar el fracaso del proyecto de TCCAx: TCCHe es una teoría próspera porque es la que provee explicaciones físicas genuinas y de donde proviene todo el éxito empírico de TCC (Wallace 2011). Otros han visto que TCCAx ha sido exitosa, particularmente para investigación filosófica y de fundamentos (Fraser 2009). Finalmente, otros han considerado que TCCHe y TCCAx son empresas complementarias y que deberían desarrollarse en paralelo (Miklos Redei en comunicación personal). No voy a adentrarme en esta discusión, sino que solamente señalaré que a pesar de las grandes diferencias entre TCCHe y TCCAx, e incluso entre la TCCWi y TCCAl, existen ciertos principios generales presentes en todas ellas. Algunos de estos principios, generalmente introducidos en TCCHe de manera no rigurosa y poco clara, son recogidos por los axiomas de TCCWi y TCCAl. Mi argumentación se centrará en uno de ellos que restringe el espectro de energía a valores positivos. Es este principio el que articula la clasificación de Wigner, el tipo de estados que existen de acuerdo a la teoría en función de la dirección temporal escogida. Pasaré a presentar este principio tal como aparece en TCCHe y TCCAx (tanto en TCCWi como en TCCAl). 


\subsection{Asimetría temporal en TCCAx}

Recordemos que una de las maneras de plantear el problema de la flecha del tiempo consiste en poner de manifiesto que, quien pretenda establecer una flecha del tiempo con significado físico, deberá buscar el modo de romper la simetría entre los miembros de los pares $T$-simétricos que resultan de la $T$-invariancia de una ley física. Naturalmente, si la teoría fuese no- $T$-invariante, sólo tendría soluciones en un sentido temporal, y la flecha del tiempo surgiría de manera inmediata. El problema con la aparición de pares $T$-simétricos, en principio, es que no parece haber argumentos no meramente arbitrarios o convencionales que permitan seleccionar una única solución como la que se considera físicamente significativa por describir una evolución en la dirección temporal pasado-a-futuro.

Si esta formulación del problema se aplica a TCCA, el argumento resulta ser el siguiente:

1. Si TCCAx es una teoría temporalmente simétrica, entonces las soluciones se presentan en pares $T$-simétricos y no es posible escoger de manera no-arbitraria uno de los miembros del par.

2. Si las soluciones se presentan en pares $T$-simétricos y no es posible escoger de manera no-arbitraria uno de los miembros del par, el espectro del operador energía-momento $P^{\mu}$ debe incluir auto-valores $p^{\mu}$ que satisfacen tanto $p^{0}>0$ como $p^{0}<0$. En términos geométricos, el espectro del operador se encontrará tanto en el semicono de luz futuro como en el pasado.

3. Pero 2 no puede darse, porque uno de los axiomas que definen TCCAx establece explícitamente que el espectro del operador de energía momento $P^{\mu}$ se encuentra confinado en el semicono de luz futuro.

4. Por lo tanto, las soluciones de la TCCAx no se presentan en pares $T$-simétricos y es posible escoger de manera no-arbitraria uno de los miembros del par.

5. Ergo, TCCAx es una teoría temporalmente asimétrica.

La validez de la premisa 3 es lo que permite dar un argumento sustancial para hacer una distinción entre una dirección del tiempo y otra. En TCCWi tal premisa es el axioma de "positividad de energía", mientras que en TCCAl es usualmente denominado "axioma de condición de espectro" (ver Bogoliubov et al. 1975, Haag 1996). Tal axioma está plausiblemente inspirado en una intuición física básica ya presente en mecánica cuántica no relativista: el espectro de energía del Hamiltoniano no puede incluir valores negativos. ${ }^{5}$ La intuición probablemente esté basada en que la materia sería altamente inestable si el espectro de energía pudiese adoptar valores negativos. Otra forma de expresar este punto es diciendo que todo Hamiltoniano tiene que tener una cota inferior, lo cual queda expresado en postular la existencia únicamente de 
Hamiltonianos acotados.

En el caso de TCCAx, tal axioma establece que $P_{0} \geq 0, P_{0}^{2} \geq 0$. Más allá de introducirse como un axioma restringiendo el espectro de energía, también naturalmente prohíbe que existan vectores de estado en la región del semicono de luz pasado $\left(C_{-}\right)$. Y este resultado se vincula directamente con la asimetría temporal. Recordemos que una manera de establecer una dirección temporal de manera no arbitraria consiste en poder escoger mediante algún recurso teórico uno de los pares $T$-simétricos que se siguen de la $T$-invariancia de las ecuaciones de la teoría. El axioma de la positividad de energía establece que frente al par simétrico $P^{0}>0, P^{0}<0$ el segundo resulta imposible desde los fundamentos axiomáticos mismos de la teoría. Su admisión implicaría que uno podría obtener valores para el operador de energía-momento en el semi-cono de luz pasado $\left(C_{-}\right)$. Confinar los valores posibles del operador energíamomento $P^{\mu}$ al semi-cono de luz futuro $\left(C_{+}\right)$correlaciona la asimetría temporal con una asimetría física fundamental de acuerdo a TCCAx expresable en términos de qué tipo de vectores son físicamente relevantes en un espacio-tiempo de Minkowski.

Vale la pena aclarar, y remarcar, que TCCAx (en sus dos versiones) es una teoría $T$-invariante en la medida en que sus ecuaciones fundamentales permanecen simétricas ante la aplicación del operador de inversión temporal (anti-linear y anti-unitario) $T$. Esto implica, según lo dicho en la Sección 2, que la estructura espacio-temporal de TCCAx es temporalmente simétrica. Sin embargo, tal como se discutió en tal sección, esto no implica que la teoría carezca de recursos teóricos para distinguir la dirección pasado-futuro de la dirección futuro-pasado, ni que tales recursos sean de naturaleza empírica o puramente fenomenológica: la no- $T$-invariancia es una condición suficiente pero no necesaria para establecer una flecha del tiempo. Como se ve, la teoría en sus aspectos estructurales (es decir, en el conjunto de sus axiomas) incluye ciertos principios que permiten establecer una asimetría temporal al poder seleccionar uno de los pares $T$-simétricos producto del carácter $T$-invariante de sus ecuaciones fundamentales. Obviamente, sin tal axioma (que re-establecería la simetría) estaríamos lidiando con una teoría diferente. Este resultado, si bien no tiene un correlato en la estructura espacio-temporal de la teoría, sí tiene un correlato en su ontología, tal como se mostró en la clasificación de representaciones irreducibles de Wigner y resulta mucho más evidente en TCCHe.

\subsection{Asimetría temporal en TCCHe}

El argumento en favor de la asimetría temporal de TCCHe funciona de la misma manera que en TCCAx aunque, naturalmente, sin apelar a un axioma. Como mencioné anteriormente, la intuición física que el espectro de energía debe sólo incluir valores positivos ya está presente en mecánica cuántica no relativista al exigir que el Hamiltoniano tenga siempre una cota inferior. En el caso de TCCHe, la tercera premisa 
del argumento anterior queda reemplazada por el análisis de las representaciones irreducibles de Wigner:

3. Pero 2 no puede darse porque los autovalores $p^{\mu}$ del operador de energíamomento $P^{\mu}$ que corresponden a valores $p^{0}<0$ (uno de los miembros del par $T$-simétrico) no refieren a estados de sistemas existentes, sobre la base de la clasificación de representaciones irreducibles de Wigner (en particular, la clase que señalamos como (ii)).

Recordemos que la clasificación de representaciones irreducibles de acuerdo al Grupo de Poincaré conduce a seis tipos de cuadrivectores de energía-momento $P^{\mu}$. De estos seis, se considera que sólo tres tienen significado físico, y son los que, precisamente, acuerdan con el axioma de la positividad de la energía de TCCAx. Lo que allí se prohíbe en términos de una condición al espectro de valores posibles del operador de energía-momento, aquí (en TCCHe) queda expresado en términos de qué clase de representaciones irreducibles son consideradas físicamente relevantes por la teoría. Y esto, nuevamente, introduce una manera de romper con el par $T$-simétrico $P^{0} \geq 0, P^{0} \leq 0$. Es decir, aunque la teoría sea $T$-invariante en sentido estricto, existe también un criterio no-arbitrario en TCCHe para seleccionar uno de los miembros del par $T$-simétrico: clases de representaciones irreducibles dadas por $P^{0} \geq 0$ no resultan en absoluto físicamente equivalentes a clases con $P^{0} \leq 0$; tal criterio está basado en el análisis de Wigner de la relación entre ontología y las simetrías de TCC; criterio que, además, es independiente y previo a los fenómenos de decaimiento de partículas subatómicas.

Como en el caso de TCCAx, esta asimetría temporal no implica que la estructura del espacio-tiempo en sí misma sea temporalmente asimétrica; sino que, sobre una base temporalmente simétrica, la teoría dispone de recursos teóricos estructurales para seleccionar, no obstante, una dirección temporal y romper así con la simetría temporal. Tanto en TCCAx como en TCCHe los recursos gravitan alrededor de cómo se determinan los valores posibles del espectro de energía del operador de energíamomento, su interpretación ontológica y su interpretación geométrica en términos del espacio-tiempo de Minkowski.

\subsection{Violación de simetrías y teorema CPT: algunas consideraciones}

Volvamos a los argumentos que apelan al decaimiento del mesón $K_{0}$ como la clave para establecer una flecha del tiempo a nivel de la física fundamental. En esta última subsección, presentaré algunas consideraciones acerca de los supuestos de la argumentación sobre los cuales, creo, es necesario reflexionar con mayor detenimiento en relación al problema de la flecha del tiempo en física. 
Como señalamos, la base fundamental del argumento es el teorema CPT, según el cual cualquier TCC relativista (i.e. invariante bajo el grupo de Lorentz) debe ser invariante ante la composición de la conjugación de la carga (C), la inversión de paridad (P) y la inversión temporal (T). En 1964, James Cronin y Val Fitch descubrieron que un tipo específico y extraño de partículas - los kaones o mesones $K_{0}$ - cuyo decaimiento violaba la simetría CP y, dado el teorema CPT, debía también violar T. A partir de ese momento, muchas posiciones acerca de la flecha del tiempo adoptaron el decaimiento de los mesones $K_{0}$ como una evidencia fuerte de asimetría temporal en TCC.

Sin embargo, más allá de los argumentos que hemos dado en las dos subsecciones anteriores que apuntaban a mostrar que TCC es temporalmente asimétrica por motivos previos e independientes de consideraciones acerca del teorema CPT y a los fenómenos de decaimiento, creo que, al menos, tienen que hacerse dos consideraciones acerca de los supuestos que el "argumento kaónico" presenta.

En primer lugar, se asume que el teorema CPT es correcto. Si bien es cierto que cuenta con una enorme evidencia empírica en su favor, la naturaleza del teorema CPT es, al menos, extraña y poco comprendida. Hilary Greaves (2010) afirma al respecto: "a pesar de la importancia del teorema CPT en física de partículas, el resultado mismo no es generalmente bien entendido" (Greaves 2010, p.72). Un primer punto podría consistir en preguntarse: ¿cómo una simetría particular (el Grupo de Lorentz) implica otra simetría (CPT)? (Greaves 2010, p.73). Una segunda cuestión atañe a la composición misma de CPT, ¿cómo puede existir una relación tan íntima entre un par de simetrías espacio-temporales como la inversión temporal y de paridad, y una simetría de una naturaleza muy distinta como es la conjugación de la carga? (Friedman 1983, Earman 1989, Greaves 2010). En el caso particular del "argumento kaónico", dado que PT son simetrías espacio-temporales pero no lo es $\mathbf{C}$, ¿cómo la heterogénea ruptura de la simetría $\mathbf{C P}$ implica la ruptura de la simetría $\mathbf{T}$ ?

En segundo lugar, también podría ponerse en tela de juicio el alcance del teorema CPT. Roger Penrose (2004) duda que CPT y la ruptura de CPT pueda extenderse a teorías que no presupongan una variedad espacio-temporal plana. Por ejemplo, afirma Penrose: "este teorema [CPT] no es inmediatamente aplicable a una teoría que presupone un espacio-tiempo curvado como el de la relatividad general de Einstein. Una de las premisas del teorema CPT es que el espacio-tiempo de trasfondo es el espacio-tiempo plano de Minkowski”. (Penrose 2004, p.818). Esto impugna - o, al menos, impone un obstáculo considerable - a cualquier intento que intente establecer una flecha del tiempo a nivel cosmológico tomando como argumento los procesos de decaimiento débil que involucran el teorema CPT.

Finalmente, volviendo a considerar los argumentos que hemos ofrecido en favor de la asimetría temporal de TCC, creo que - al menos para la discusión sobre la flecha del tiempo - el papel que juega el teorema CPT y sus extrañas y espo- 
rádicas violaciones, es poco relevante. ¿Es la ruptura de la simetría $\mathbf{T}$ dada por el decaimiento del kaón neutro aquello que distingue de manera no convencional entre las direcciones temporales en TCC? La respuesta no es completamente clara. Las incidentales violaciones de $\mathbf{T}$ por parte de las interacciones débiles son sólo eso: fenómenos incidentales. Si tales fenómenos no existiesen, TCC sería, de todas maneras, una teoría temporalmente asimétrica ya que, a partir de postulados propios de la teoría, se dispone de un criterio físico y no-arbitrario para seleccionar uno de los pares $T$-simétricos, es decir, a pesar del carácter $T$-invariante de sus leyes dinámicas.

\section{Conclusiones}

Las discusiones en torno al problema de la flecha del tiempo siguen presentes en nuestros días y continúan generando grandes controversias tanto en filosofía como en los fundamentos de la física. Las distintas respuestas al problema, así como las variadas formulaciones del problema mismo, abren un gran abanico de líneas de investigación. Este trabajo se focalizó en las recientes discusiones sobre el problema de la flecha del tiempo en el terreno de TCC. Como he mostrado, TCC posee una estructura teórica que no es temporalmente simétrica de manera absoluta y que permite distinguir, de manera no arbitraria, la dirección pasado-a-futuro de la dirección futuro-a-pasado. Tal distinción puede llevarse a cabo, como he mostrado, a pesar de que la teoría es $T$-invariante.

Sin embargo, hay algunos supuestos que no han sido cuestionados aquí. No hemos considerado, por ejemplo, el alcance final de la propiedad de $T$-invariancia de las ecuaciones dinámicas: ¿es una condición necesaria y suficiente para la flecha del tiempo? ¿O, como he asumido a lo largo del artículo, es una condición meramente suficiente? Algunos autores, como Maudlin (2002), que defienden una especie de primitivismo respecto de la flecha del tiempo parecen pensar una hipotética no- $T$ invariancia de las leyes físicas es meramente una condición suficiente, pero no necesaria. Mario Castagnino y Olimpia Lombardi (2009) parecen seguir una línea similar al presentar un enfoque puramente geométrico de la flecha del tiempo que no recae en la noción de invariancia temporal.

Sin embargo, ¿por qué las leyes dinámicas no reflejarían una asimetría que, de una u otra manera, está presente en la teoría misma? Si la asimetría es puramente de facto (por ejemplo, debida a ciertas condiciones iniciales), la razón es clara. Pero el argumento que he presentado en este artículo y las posiciones citadas en el párrafo anterior parecen ser más fundamentales que una mera asimetría factual: la distinción entre ambas direcciones temporales puede hacerse mediante recursos estructurales de la teoría y es válida en todas sus formulaciones y modelos. Si las simetrías de la dinámica de una teoría permiten conocer las simetrías de, por ejemplo, su estructura 
espacio-temporal (North 2009), ¿por qué la identificación de una asimetría temporal fundamental no tiene un correlato directo con una asimetría en las leyes dinámicas y en la estructura espacio-temporal? Esto nos lleva nuevamente a una pregunta formulada anteriormente: ¿sólo leyes no- $T$-invariantes permiten fundamentar una flecha del tiempo en un sentido robusto? Como he mostrado a lo largo de este trabajo, creo que la respuesta es negativa y he ofrecido una estrategia alternativa. Sin embargo, no es claro - al menos para mí - cuál es el alcance y límite de la noción de invariancia temporal bajo estas consideraciones, y quedaría como materia de investigación futura.

Por otro lado, desplazando el terreno de la discusión a la teoría cuántica de campos, se han abierto nuevos interrogantes. En primer lugar, si bien hemos argumentado que se trata de una teoría temporalmente asimétrica debido a postulados internos de la teoría, no hemos ofrecido ninguna justificación externa a la teoría de tal asimetría. De hecho, tanto la mecánica cuántica estándar como la teoría especial de la relatividad son teorías temporalmente simétricas. Podríamos preguntarnos si acaso existe alguna justificación para que TCC sea una teoría temporalmente asimétrica, sin apelar a razones de exigencia teórica propia. Finalmente, TCC pone al descubierto la estrecha relación entre el grupo de simetría de una teoría, su ontología y sus consideraciones temporales: creemos que éste es un terreno sumamente prometedor para la investigación filosófica y científica sobre el tiempo y su naturaleza.

\section{Referencias}

Albert, D. Z. 2001. Time and Chance. Cambridge, MA: Harvard University Press.

Arntzenius, F. 2012. The CPT theorem. In: C. Callender (ed.) The Oxford Handbook of Philosophy of Time, pp.634-46. Oxford: Oxford University Press.

Bain, J. 2011. Quantum field theories in classical spacetimes and particles. Studies in History and Philosophy of Modern Physics 42: 98-106.

Brading, K.; Castellani, E. 2007. Symmetries and invariances in classical physics. In: J. Butterfield; J. Earman (eds.) Philosophy of Physics. Part B, pp.1331-67. Amsterdam: Elsevier.

- 2017. Symmetry and Symmetry Breaking. In: E. N. Zalta (ed.) The Stanford Encyclopedia of Philosophy. Fall edition 2008. http://plato.stanford.edu/archives/fall2008/ entries/symmetry-breaking/. Acceso: 04/01/2018.

Bogoliubov, N.; Logunov, A. A.; Todorov, I. T. 1975. Axiomatic Quantum Field Theory. Reading: Benjamin- Cummings.

Castagnino, M.; Lombardi, O. 2004. The generic nature of the global and non-entropic arrow of time and the double role of the energy-momentum tensor. Journal of Physics A (Mathematical and General) 37: 4445-63.

- 2005. A global and non-entropic approach to the problem of the arrow of time. In: A. Reimer (ed.) Spacetime Physics Research Trends. Horizons in World Physics, pp.74-108. New York: Nova Science. 
2009. The global non-entropic arrow of time: from global geometrical asymmetry to local energy flow. Synthese 169: 1-25.

Earman, J. 1989. World enough and space-time. Cambridge, MA: MIT Press.

Fraser, D. 2011. How to take particle physics seriously: A further defense of the axiomatic quantum field theory. Studies in History and Philosophy of Modern Physics (B) 42: 126-35.

Friedman, M. 1983. Foundations of space-time theories. Princeton: Princeton University Press.

Greaves, H. 2010. Towards a Geometrical Understanding of the CPT Theorem. The British Journal of Philosophy of Science 61(1): 27-50.

Haag, R. 1996. Local Quantum Physics. Fields, Particles, Algebras. Berlin: Springer

Horwich, P. 1987. Asymmetries in Time. Cambridge, MA: MIT Press.

Kuhlmann, M.; Wayne, A. 2002. Ontological aspects of Quantum Field Theory. Singapore: World Scientific.

Kuhlmann, M. 2010. The Ultimate Constituents of the Material World: In Search of an Ontology for Fundamental Physics. Frankfurt: Ontos Verlag.

- 2012. Quantum Field Theory. In: E. N. Zalta (ed.) The Stanford Encyclopedia of Philosophy. Winter edition 2012. http://plato.stanford.edu/archives/win2012/entries/ quantum-field-theory/, Acceso: 20/07/2017

McTaggart, J. M. E. 1908. The Unreality of Time. Mind 17: 457-73.

Maudlin, T. 2002. Remarks on the passing of time. Proceedings of the Aristotelian Society 102: 237-52.

- 2007. Metaphysics within physics. New York: Oxford University Press.

North, J. 2009. Two views on time reversal. Philosophy of Science 75: 201-23.

- 2012. Time in Thermodynamics. In: C. Callender (ed.) The Oxford Handbook of Philosophy of Time, pp.312-50. Oxford: Oxford University Press.

Penrose, R. 2004. Road to reality. London: Jonathan Cape.

Price, H. 1996. Time's Arrow and Archimedes' point: new directions for the physics of time. New York: Oxford University Press.

- 2012. The flow of time. In: C. Callender (ed.) The Oxford Handbook of Philosophy of Time, pp.276-311. Oxford: Oxford University Press.

Ruetsche, L. 2011. Interpreting Quantum Theories. Oxford: Oxford University Press.

Sachs, R. 1987. The Physics of Time Reversal. London: University Chicago Press.

Savitt, S. 1996. The direction of time. The British Journal for the Philosophy of Science 47(3): 347-70.

Sklar, L. 1974. Space, time and spacetime. Berkeley: University of California Press

Streater, R. F; Wightman, A. 1964. PCT, Spin and Statistics and All That. Princeton: AdissonWesley.

Wallace, D. 2011. Taking particle physics seriously: A critique of the algebraic approach to quantum field theory. Studies in History and Philosophy of Modern Physics (B) 42: 116-25.

- 2012. The arrow of time in physics. In: A. Bardon; H. Dyke (eds.) A Companion to the Philosophy of Time, pp.262-81. Wiley-Blackwell.

Weinberg, S. 1987. Elementary Particles and the Laws of Physics, The 1986 Dirac Memorial Lectures. Cambridge: Cambridge University Press.

Wigner, E. 1939. On unitary representations of the inhomoneneous Lorentz group. Annals of Mathematics 40: 149-204. 


\section{Notas}

${ }^{1}$ Vale la pena aclarar el significado del término 'objetivo' en este contexto. Usualmente, el término refiere a algo que resulta independiente de la mente o de un sujeto cognoscente: una propiedad adjudicada a algún objeto no resulta como producto de nuestras creencias o estructuras cognoscitivas. Sin embargo, en filosofía de la física el término no ha de entenderse como independiente de un sistema de referencia: una enorme cantidad de descripciones físicas dependen del sistema de referencia y no por eso son consideradas como puramente epistémicas o no objetivas. En el caso de la flecha del tiempo en un contexto relativista, por ejemplo, el caso es análogo: en ciertas condiciones sistemas de referencia separados por una relación de tipo espacio podrían desacordar respecto de la dirección temporal de una serie de eventos. Sin embargo, ello no debiera afectar la objetividad de la dirección temporal relativa a un sistema de referencia. Lo importante en este contexto es que dentro de un sistema de referencia (cualquiera) sea posible definir una dirección temporal de manera no arbitraria. Si cualquier sistema de referencia puede lograr tal objetivo (más allá de que en ciertos casos diferentes sistemas de referencia puedan desacordar respecto de cuál es la direccionalidad), es posible establecer una flecha objetiva del tiempo relativa a un sistema de referencia. Uno no dispondría de argumentos físicos para una flecha del tiempo si dentro de un sistema de referencia, no fuera posible establecer una flecha del tiempo (y esto se pudiese replicar en cualquier sistema de referencia). Nótese que el término contrario a objetivo es "subjetivo" y no "relativo a...". Agradezco a un referí anónimo por señalar la necesidad de este comentario.

${ }^{2} \mathrm{Si}$ bien pueden trazarse ciertas diferencias conceptuales entre las nociones de "asimetría temporal", "flecha del tiempo" y "dirección del tiempo", en este trabajo las utilizaré indistintamente: la idea central condensada en estas expresiones es que el tiempo exhibe una asimetría entre pasado y futuro, y que la dirección pasado-futuro es diferente a la dirección futuro-pasado. Aunque es cierto que una asimetría no implica una direccionalidad (aunque la contrapuesta si es cierta), en la mayoría de los casos donde podemos encontrar una asimetría temporal, podemos convencionalmente adjudicar una dirección de manera unívoca: nada en la asimetría nos dice cuál es la dirección pasado-futuro, pero sí nos dice que la dirección pasado-futuro no es igual que la dirección futuro-pasado.

${ }^{3}$ Esta sección está basada, fundamentalmente, en Kuhlmann (2010, pp.87-93) con algunas adaptaciones.

${ }^{4}$ En palabras del propio Wightman: "la teoría cuántica de campos nunca alcanzó un estadio en el cual uno pueda decir que estaba libre de contradicciones internas, ni al revés. De hecho, el principal problema de la teoría cuántica de campos resultó ser que o bien había que destruirla o curarla, es decir, o se mostraba que las idealizaciones involucras en las nociones fundamentales de la teoría eran incompatibles en sentido físico, o la teoría se moldeaba nuevamente de tal manera que provea un lenguaje práctica para la descripción de la dinámica de partículas elementales" (Streater y Wightman 1964, Introduction).

${ }^{5}$ Curiosamente, esta condición es uno de los motivos por los cuales al definir la operación de inversión temporal en mecánica cuántica no relativista y relativista se suele apelar a un operador anti-unitario que no sólo invierte la dirección temporal $\left(T t T^{-1}=-t\right)$, sino también $\left(T z T^{-1}=z \star \iota\right)$, lo cual conduce a que el Hamiltoniano transforme ante inversión temporal como $\left(\right.$ THT $\left.^{-1}=H^{\iota}\right)$ 


\section{Agradecimentos}

Agradezco al Grupo de Filosofía de las Ciencias de la Universidad de Buenos Aires (Facultad de Ciencias Exactas y Naturales) por el constante apoyo y la motivación intelectual. En particular, agradezco a Olimpia Lombardi por sus comentarios a una primera versión de este artículo. También agradezco a dos referís anónimos: el artículo fue mejorado sustancialmente a la luz de sus comentarios y sugerencias. Este artículo fue posible gracias a una Beca Doctoral de CONICET (Argentina) y en el marco del Proyecto John Templeton Foundation (57919). 